\title{
Analog Piezoelectric-Driven Tunable Gratings With Nanometer Resolution
}

\author{
Chee Wei Wong, Member, IEEE, Member, ASME, Yongbae Jeon, George Barbastathis, Member, IEEE, and \\ Sang-Gook Kim, Member, IEEE, Member, ASME
}

\begin{abstract}
This paper presents the design, fabrication, and characterization of a piezoelectrically actuated MEMS diffractive optical grating, whose spatial periodicity can be tuned in analog fashion to within a fraction of a nanometer. The fine control of the diffracted beams permits applications in dense wavelength-division multiplexing (DWDM) optical telecommunications and high-resolution miniaturized spectrometers. The design concept consists of a diffractive grating defined on a deformable membrane, strained in the direction perpendicular to the gratings grooves via thin-film piezoelectric actuators. The tunable angular range for the first diffracted order is up to $400 \mu \mathrm{rad}$ with $0.2 \%$ strain ( $\sim 8 \mathrm{~nm}$ change in grating periodicity) at $10 \mathrm{~V}$ actuation, as predicted by device modeling. The actuators demonstrate a piezoelectric $d_{31}$ coefficient of $-100 \mathrm{pC} / \mathrm{N}$ and dielectric constant $\varepsilon_{\mathrm{r}}$ of 1200. Uniformity across the tunable grating and the out-of-plane deflections are also characterized and discussed.

[1036]
\end{abstract}

Index Terms-Analog, deformable membrane, diffractive gratings, nanometer resolution, thin-film piezoelectric, tunable.

\section{INTRODUCTION}

$\mathbf{O}$ PTICAL MEMS have included many innovative devices, such as micromirrors for beam steering in projection displays and optical crossconnects, tunable Fabry-Pérot cavities for variable wavelength vertical cavity surface emitting lasers, and gratings with tunable dispersion, among many others. Our device is a tunable diffractive grating, with fine analog control over the grating period for reconfiguration in optical filters. Immediate applications include tunable wavelength multiplexers/demultiplexers, external cavity tunable lasers, active compensation for thermal disturbances to optical instruments involving gratings, and miniaturized spectrometers. We refer to the state-of-the-art tunable gratings or binary optical devices as "digital": for example, the Polychromator [1], the Grating Light Valve [2], the MEMS Compound Grating [3], or a liquid-crystal phased-array with addressable electrodes for beam steering [4]. The conceptual designs of these devices involve vertical (out-ofplane) control of the gratings beams or phase segments via electrostatic actuation to alter the optical response. The resolution,

Manuscript received November 6, 2003; revised February 11, 2004 This work was supported by the ASML Holding N. V. Corporation. Subject Editor G. Fedder.

C. W. Wong was with the Department of Mechanical Engineering, Massachusetts Institute of Technology, Cambridge, MA 02139 USA. He is now with Columbia University, New York, NY 10027 USA (e-mail: cww2104@ columbia.edu).

Y. Jeon, G. Barbastathis, and S.-G. Kim are with the Department of Mechanical Engineering, Massachusetts Institute of Technology, Cambridge, MA 02139 USA.

Digital Object Identifier 10.1109/JMEMS.2004.839592

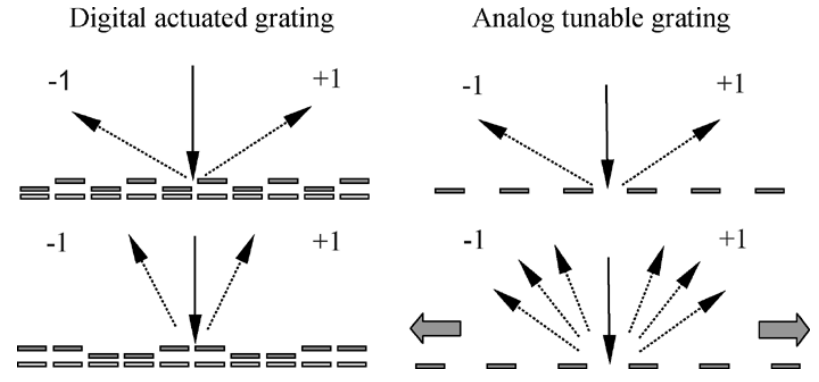

Fig. 1. Actuation concept of analog and digital tunable gratings. In-plane actuation permits analog control over the diffracted angles.

however, is limited by the beam width (on the order of microns), or the electrode width of the liquid-crystal phased-array.

Analog tunability, which permits finer control over digital tunability, on diffractive gratings has been reported via a few mechanisms, as listed in the following: 1) thermal actuation with reported diffraction angle modulation from 1 to 35 mradians [5], [6], 2) acoustic modulation to scan the transmitted optical beam angle [7], 3) magnetic and proof mass actuation on fiber Bragg gratings [8], [9], and 4) electrostatic MEMS actuation for variable blaze grating angles, with reported tuning up to 44 mradians [10].

Here we present a method of analog tuning to achieve tunability in the diffracted angle up to $400 \mu \mathrm{rads}$, with a resolution of $2 \mu \mathrm{rads}$, with integrated thin-film piezoelectric actuators on a deformable membrane. Compared with the digital designs, our device concept trades a large tuning range for fine angular resolution. Moreover, the use of integrated piezoelectric actuators allows fast dynamic responses in the order of $\mathrm{MHz}$, low-power consumption, low driving voltage (compared to thermal actuation), and better localization of tunability on individual sets of gratings, in contrast with the analog methodologies mentioned above. In our design, the diffraction grating period is tuned by transverse (in-plane and perpendicular to the grating grooves axis) actuation forces applied on the grating structure. This is illustrated in Fig. 1, where the grating period is increased continuously, resulting in steering of the diffracted orders [11], [12].

\section{DeVICE DEsign}

To achieve transverse actuation, we first define the grating grooves on a supporting deformable membrane. The membrane is then mechanically stretched by thin-film piezoelectric actuators, as shown in Fig. 2. The piezoelectric actuators, a lead zirconate titanate (PZT) material located at both ends of the membrane, are capable of producing sufficient force to strain the 


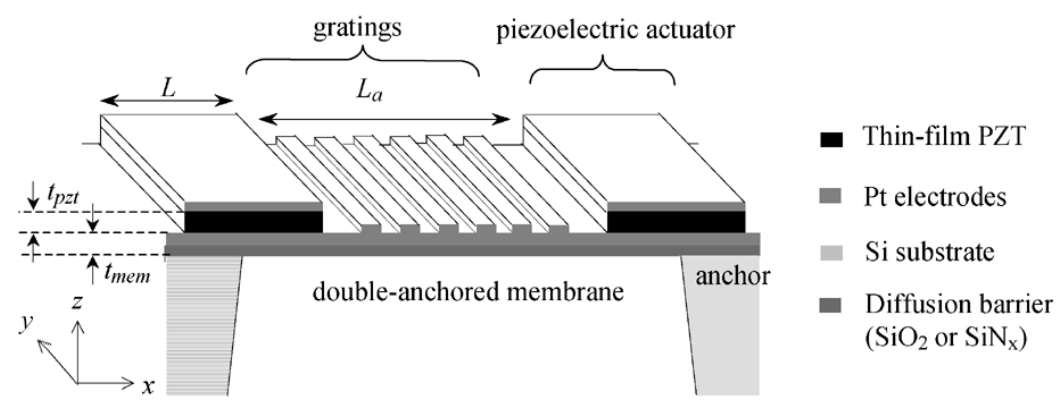

Fig. 2. Design schematic of double-anchored deformable membrane, driven via thin-film piezoelectric actuators. The gratings, defined on top of the membrane, are tuned progressively along with the membrane.

membrane up to $0.3 \%$, or equivalently, $0.3 \%$ diffracted angle change. This is accomplished through application of an electric field across the PZT film to induce strain, due to reorientation of electric polarizations. This correspondingly strains the membrane in the transverse direction and, subsequently, the $4 \mu \mathrm{m}$ period binary phase grating imprinted on the membrane. The double-anchored membrane configuration, illustrated in Fig. 2, minimizes out-of-plane displacements of the grating grooves from unbalanced film residual stresses and bending moments of the PZT under actuation. An alternative method of transverse tuning of the grating period uses electrostatic comb-drives on a more compliant grating structure [11], [12].

\section{A. Deformable Membrane Design}

The membrane in-plane displacement modeling, along the grating period axis, begins with a piezoelectric bimorph model where the membrane deformation $\delta_{x}$ is expressed as [13]

$$
\delta_{x}=\frac{d_{31} E_{\mathrm{pzt}} A V_{a}}{t_{\mathrm{pzt}} k_{x}}
$$

where $d_{31}$ is the piezoelectric coupling coefficient, $E_{i}$ is the $i^{\text {th }}$ material Young modulus, $A_{i}$ is the $i^{\text {th }}$ cross-sectional area, $V_{a}$ is the applied voltage, $t_{\mathrm{pzt}}$ is the thickness of PZT layer, $k_{x}$ is the effective axial stiffness $=\Sigma_{i} E_{i} A_{i} / L$, and $L$ is the PZT beam length along the $x$-axis. Boundary conditions are applied to reflect the double-anchored membrane structure (with symmetry across the membrane midpoint) and the solution reached iteratively. For an isotropic wide membrane [14], $E_{i} \rightarrow E_{i} /\left(1-v_{i}^{2}\right)$ and $d_{31} \rightarrow d_{31}\left(1+v_{i}\right)$, where $v_{i}$ is the material Poisson's ratio. As expected intuitively, the membrane strain increases with $L$ and with $d_{31}$ for a fixed grating size and actuation voltage. Fig. 3(a) shows the design variation against nondimensional length ratio ( $L$ normalized by grating aperture size, $L_{a}$ ) for specific design parameters. In addition, the membrane strain increases linearly with applied voltage due to the small amplitude displacement. Design parameters of our double-anchored membrane are summarized in Table I. For these specific values, the analytical model for a case of Design II predicts $187 \mathrm{~nm} x$-axis membrane displacement with a single actuator at $10 \mathrm{~V}$. This corresponds to a $5.28 \mathrm{~nm}$ grating period change and $0.13 \%$ membrane strain, assuming uniform deformation across the membrane.

We use a numerical finite-element tool (CoventorWare) as a confirmation on our analytical results. Both models are in good agreement. A transverse ( $x$-axis) displacement of $280 \mathrm{~nm}$ (for Design I) is predicted in the finite-element model, as shown in Fig. 3(b). The finite-element model predicts a maximum $y$-axis Poisson contraction of $83 \mathrm{~nm}$ across the $300 \mu \mathrm{m}$ grating, and a maximum Mises stress concentration of $220 \mathrm{MPa}$. In addition, the finite-element model provides an estimate of the membrane out-of-plane bow when actuated. The calculated maximum vertical deflection is $1.941 \mu \mathrm{m}$ at the center of a $232 \mu \mathrm{m}$ (in length) membrane for an applied voltage of $10 \mathrm{~V}$. Control of the residual stress in the membrane with a low-stress silicon nitride diffusion barrier reduces the deflection and bow resulting from actuating the membrane.

\section{B. Optical Design}

In the small angle limit, the diffracted angular change $\Delta \theta$ is related to the grating period change as

$$
\Delta \theta \cong \frac{m \lambda \Delta d}{d^{2}}
$$

where $m$ is the diffracted order, $\lambda$ is the wavelength, $\Delta d$ is the grating period change, and $d$ the grating period. For a $632.8 \mathrm{~nm}$ laser on a $4 \mu \mathrm{m}$ period grating, a $5.28 \mathrm{~nm}$ period change (for Design II at $10 \mathrm{~V}$ ) corresponds to an angular change of $209 \mu \mathrm{rads}$ for the first diffracted order. Based on the fine voltage control [15] previously demonstrated on a thin-film piezoelectric material, the resolution of the grating period change is estimated about $0.5 \AA$, corresponding to angular resolution better than $2.1 \mu \mathrm{rads}$, although the current metrology limits the observable resolution in the order of $1 \mathrm{~nm}$. Out-of-plane membrane tilt and bending, asymmetrical rotation of the membrane when actuated, thermal disturbances, modal vibrations, and optical setup and processing techniques will limit the achievable resolution.

The bow shape profile of the actuated binary phase grating, as derived in the deformable membrane mechanical design, is added as a nonuniform phase at the object plane with a finiteaperture window. At the Fourier plane [16], this is observed as a spatial spreading out of the first diffracted order energy, although the first order diffraction efficiency remains approximately unchanged at $20.3 \%$. This is illustrated in comparison with an optically flat diffraction grating in Fig. 4, wherein both cases are considered in the Fraunhofer regime. The membrane bow contributes approximately an additional $56 \%$ shift in the first diffracted order, compared to the same grating period actuation on an ideal flat grating. The additional shift needs to be 
(a)

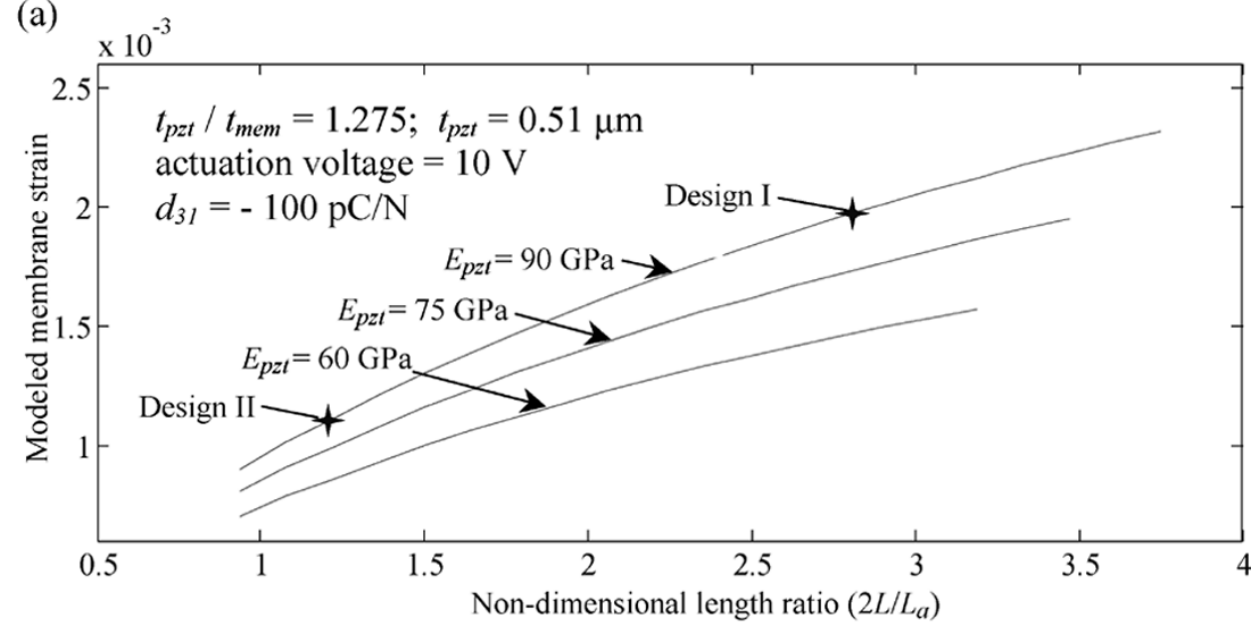

(b)
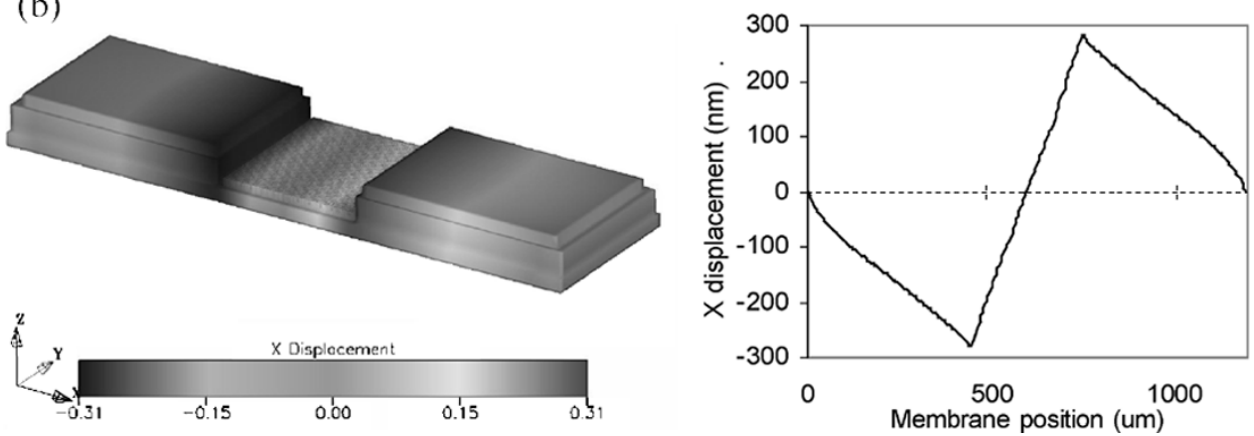

Fig. 3. (a) Design space of membrane strain against nondimensional PZT length. (b) Finite-element confirmation of design analysis. Specific result of Design I at $10 \mathrm{~V}$ illustrates $x$-axis membrane displacement. The $z$-axis is exaggerated $20 \mathrm{X}$ to show the different layers in the membrane. The units are in microns.

TABLE I

Analog Piezoelectric Tunable Grating Parameters

\begin{tabular}{|c|c|c|}
\hline Geometry & Design I & Design II \\
\hline PZT length, $L(\mu \mathrm{m})$ & 450 & 200 \\
\hline PZT thickness, $t_{p z t}(\mu \mathrm{m})$ & \multicolumn{2}{|c|}{0.51} \\
\hline grating aperture length, $L_{a}(\mu \mathrm{m})$ & \multicolumn{2}{|c|}{320} \\
\hline membrane thickness, $t_{m e m}(\mu \mathrm{m})$ & \multicolumn{2}{|c|}{0.4} \\
\hline membrane width $(\mu \mathrm{m})$ & \multicolumn{2}{|c|}{300} \\
\hline \multicolumn{3}{|l|}{ Performance } \\
\hline$d_{3 l}$ coefficient, inferred $(\mathrm{pC} / \mathrm{N})$ & \multicolumn{2}{|c|}{$-100 \pm 15$} \\
\hline$d_{33}$ coefficient, direct measurement $(\mathrm{pC} / \mathrm{N})$ & \multicolumn{2}{|c|}{275.4} \\
\hline$\varepsilon_{r}$, relative dielectric constant & \multicolumn{2}{|c|}{1200} \\
\hline minimum resolvable grating period change $(\mathrm{nm})$ & \multicolumn{2}{|c|}{$\sim 0.6$} \\
\hline grating period change $(\mathrm{nm})$ & 8.3 at $9 \mathrm{~V}$ & 4.9 at $10 \mathrm{~V}$ \\
\hline membrane strain & $0.21 \%$ at $9 \mathrm{~V}$ & $0.12 \%$ at $10 \mathrm{~V}$ \\
\hline diffracted angular change at $10 \mathrm{~V}$ ( $\mu$ radians) & 486 & 154 \\
\hline
\end{tabular}

taken into account in the optical design for specific optical telecommunication devices and miniaturized spectrometers.

\section{FABRICATION}

The microfabrication process flow, involving both surface and bulk micromachining, is shown in Fig. 5. Thermal oxide $(200 \mathrm{~nm})$ is first grown by wet oxidation, followed by plasma-enhanced chemical vapor deposition of $200 \mathrm{~nm}$ silicon nitride on the wafer backside. The nitride is patterned to form a hard-mask (for a backside $\mathrm{KOH}$ etch in the penultimate step). An alternative to these first few steps is to use low-stress liquid-plasma chemical vapor deposition (LPCVD) silicon nitride to control the residual stress in the films on the wafer frontside and to avoid nitride pinholes on the wafer backside from longer $\mathrm{KOH}$ etches. Next, a $220 \mathrm{Pt} / \mathrm{Ti}$ layer is evaporated and patterned via lift-off on the frontside as the bottom electrode for the PZT film. Sol-gel PZT is subsequently spun-on in repeated steps-each coating with $\sim 60 \mathrm{~nm}$ PZT thickness - and annealed to create a high-quality PZT film 


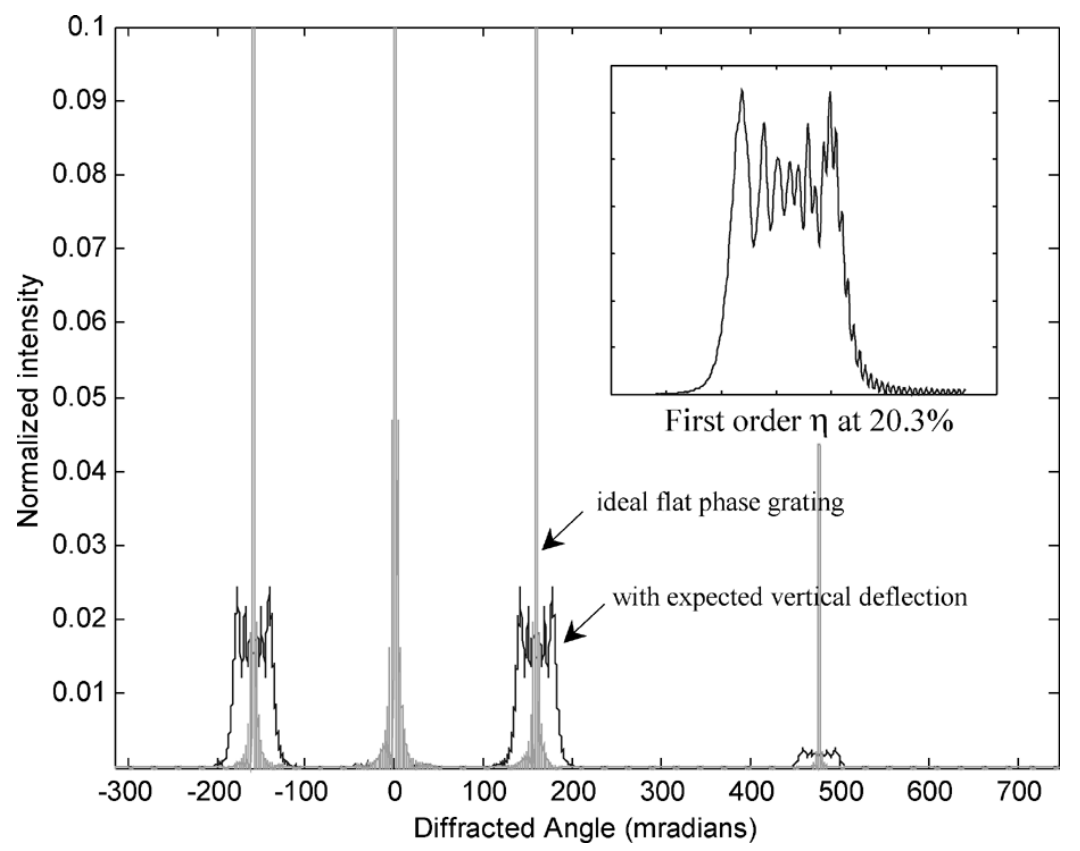

Fig. 4. Normalized intensity profile comparison between an ideal binary phase grating and one with a bow in the deformable membrane design. The duty cycle and grating step height is taken at $50 \%$ and $\lambda / 4$ respectively for an ideal grating.

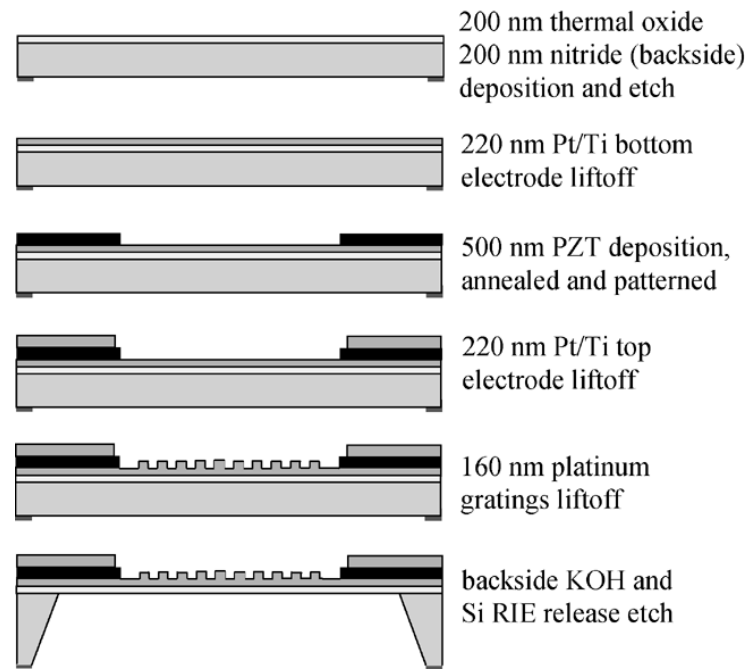

Fig. 5. Microfabrication process flow of the analog tunable grating, with surface and bulk micromachining.

with $0.5 \mu \mathrm{m}$ total thickness. PZT crack formation and hillock issues were resolved with experimentation over the specific $\mathrm{Pt} / \mathrm{Ti}$ bottom electrode and PZT fabrication conditions. The piezoelectric layer is next patterned with a wet-etch technique [17] and the top $220 \mathrm{~nm} \mathrm{Pt/Ti} \mathrm{electrode} \mathrm{defined} \mathrm{with} \mathrm{an} \mathrm{evap-}$ oration and lift-off procedure similar to the bottom electrode. The diffraction grating is then separately defined with another Pt evaporation and lift-off step to allow a grating thickness of $158 \mathrm{~nm}$ (quarter wavelength of $632.8 \mathrm{~nm}$ incident $\mathrm{HeNe}$ laser). The wafer is subsequently etched from the backside with $\mathrm{KOH}$ and the double-anchored membrane defined and released with a $5 \mu \mathrm{m}$ Si RIE from the backside.

The fabricated device is shown in Fig. 6. The completed PZT has a predominant perovskite phase, shown in Fig. 7(a), and has

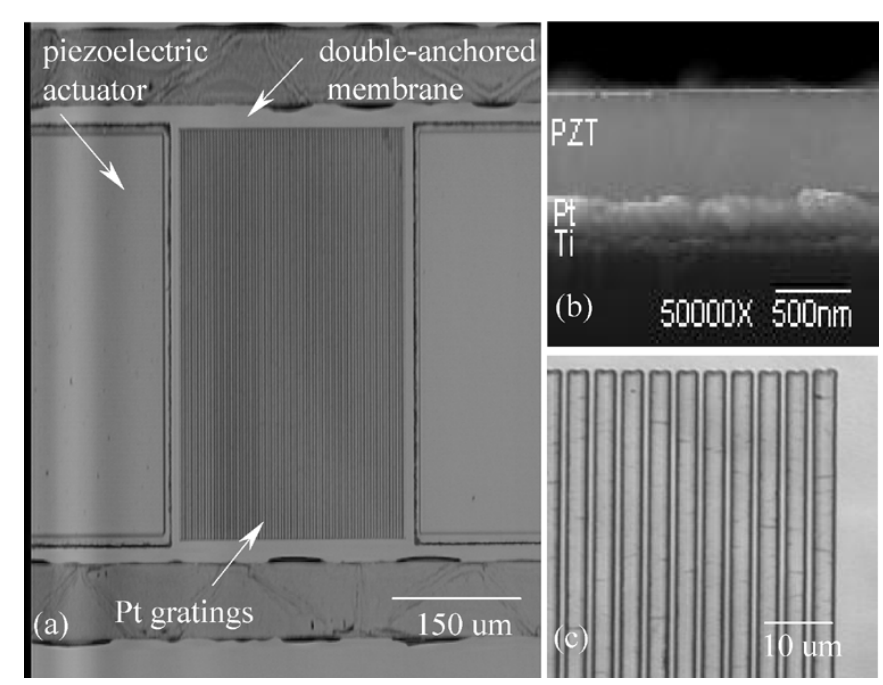

Fig. 6. (a) Piezoelectric-actuated tunable grating under $120 \mathrm{X}$ magnification. (b) SEM cross-section image of fabricated PZT actuator on $\mathrm{Pt} / \mathrm{Ti}$ electrodes. (c) Magnified view of Pt gratings with $4 \mu \mathrm{m}$ period.

an average grain size on the order of $0.1 \mu \mathrm{m}$. Ferroelectric characterization, Fig. 7(b), suggests an excellent dielectric constant $\varepsilon_{\mathrm{r}}$ of above 1200 and a dielectric loss below 0.05 . The coercive field and saturation polarization are estimated at $60 \mathrm{kV} / \mathrm{cm}$ and $67 \mu \mathrm{C} / \mathrm{cm}^{2}$ respectively as shown in Fig. 8. Fatigue cycle experiments on the piezoelectric material suggest nonstop operation above $10^{10}$ cycles under a $5-\mathrm{V} 19.6-\mu$ s rectangular pulse-train signal with an approximate 15\% decrease in PZT performance [18]. The PZT performance also quickly recovered after the first test, and also operated above $10^{10}$ cycles in the second test cycle. For longer continuous operation, the PZT could be operated with slower pulses, compared to the $19.6-\mu$ s pulse times used in our fatigue cycle experiments. The power consumption of the PZT film at $10 \mathrm{~V}$ actuation is gauged at $30 \mathrm{nW}$. The fabricated 
(a)
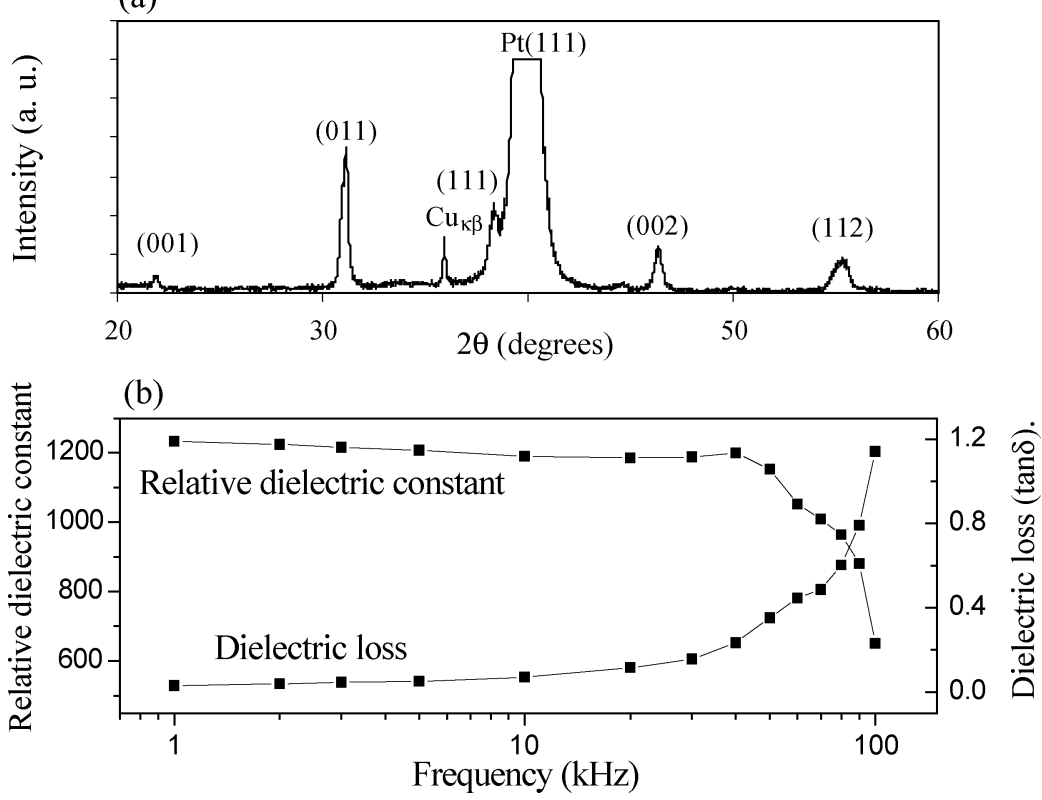

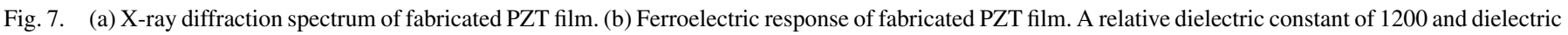
loss of less than 0.05 was measured.

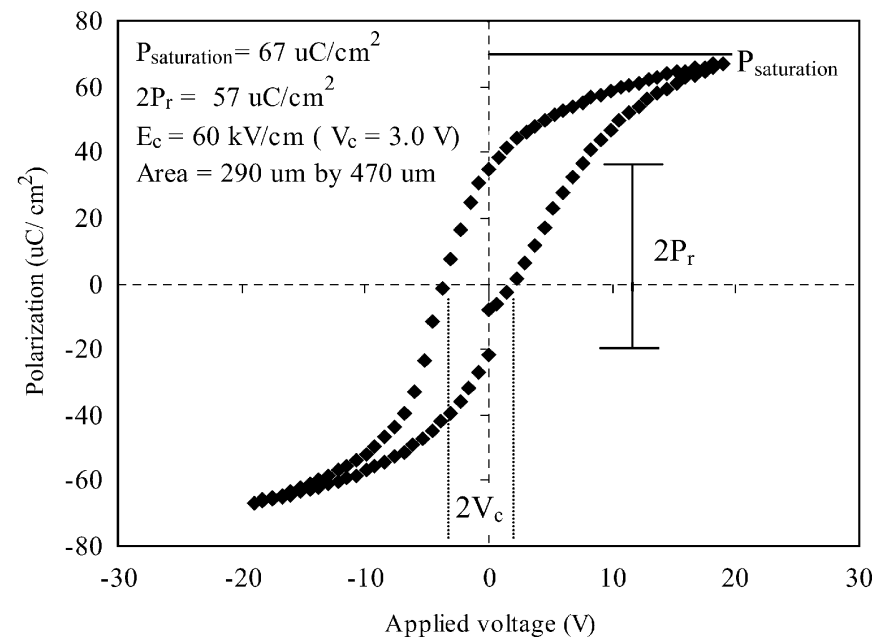

Fig. 8. Hysteresis characterization of piezoelectric film after completion of device fabrication.

binary phase grating also has a measured duty cycle varying between $42 \%-66 \%$, depending on the process conditions. First-order diffraction efficiency was measured at 7.6\%, respectively, for the fabricated grating (without actuation), although higher efficiencies closer to the theoretical $40.5 \%$ could be achieved with better process control [19].

\section{DEVICE Characterization}

The membrane strain deformation by the thin-film PZT actuators is measured with a Computer Microvision instrument [20]. This instrument reconstructs three-dimensional (3-D) images of microscopic targets using the optical sectioning property of a light microscope and postprocesses the combined images to analyze the target motion with nanometer precision. For optical tests, the device is either packaged by wire-bonding onto a pin

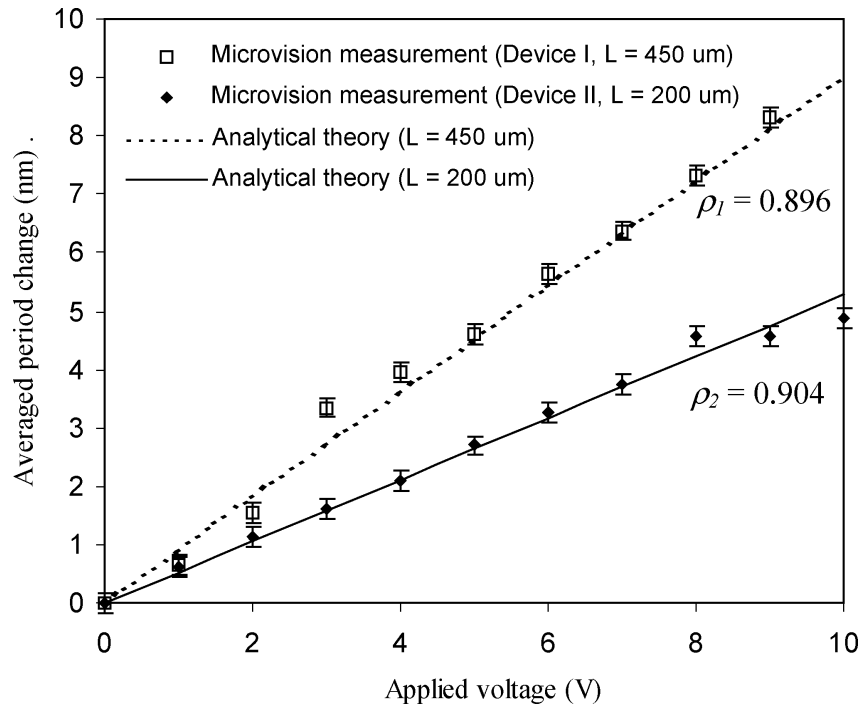

Fig. 9. Measured period change against applied voltage for two different device designs [12]. Both results match with the analytical model for a single set of material properties and with a single fitted $d_{31}$ coefficient at $-100 \mathrm{pC} / \mathrm{N}$.

grid array chip or mounted on a probe station with a modified optical setup.

\section{A. Membrane Deformation Results}

Measurement of a device fabricated according to Design I (450 $\mu \mathrm{m}$ PZT length) demonstrates a $229 \pm 2 \mathrm{~nm}$ total membrane displacement at $9 \mathrm{~V}$. By tracking the displacements of individual grating beams, an average period change of $8.3 \mathrm{~nm}$ $(0.21 \%$ membrane strain) at $9 \mathrm{~V}$ actuation is observed. This corresponds to an expected diffracted angular change of $328 \mu \mathrm{rads}$ for our fabricated device parameters. A device with Design II (200 $\mu \mathrm{m}$ PZT length) parameters shows a 4.9-nm period change at $10 \mathrm{~V}$ (corresponding to $0.12 \%$ membrane strain and an expected $194 \mu$ radians diffracted angle change). The 


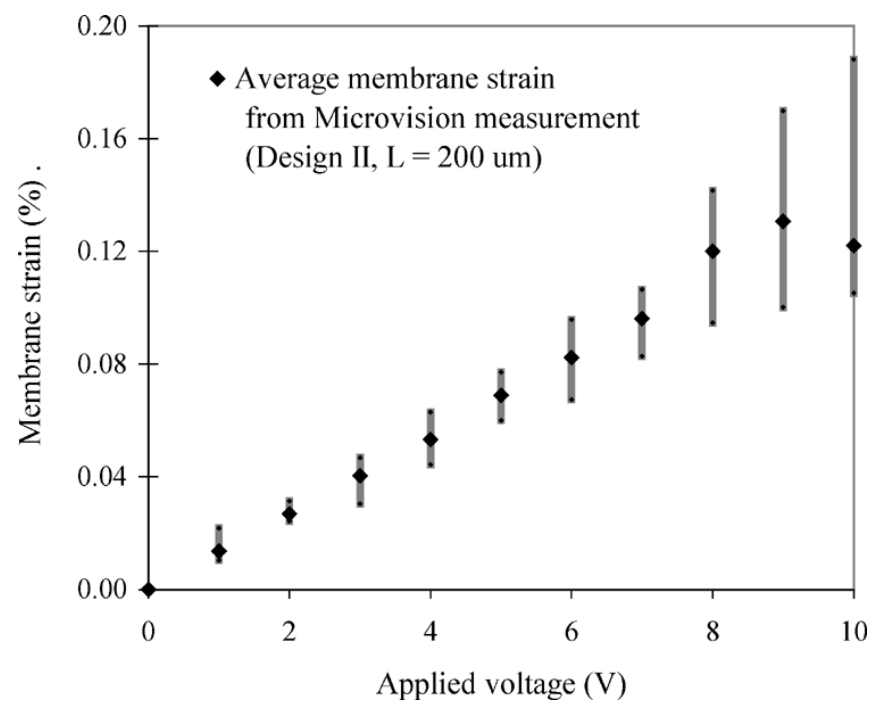

Fig. 10. Uniformity of membrane strain under actuation. A uniformity variation of $16 \%$ (defined as the standard deviation over the averaged value) is measured. The error bars depict the maximum and minimum values of the measurements.

period change at $1 \mathrm{~V}$ for both devices is approximately $0.6 \mathrm{~nm}$, calculated from total membrane displacement. As summarized in Fig. 9, both experimental device measurements are in good agreement (correlation coefficient $\rho \sim 0.9$ ) with the single analytical formulation for a $d_{31}$ coefficient of approximately $-100 \pm 15 \mathrm{pC} / \mathrm{N}$. A consistent set of material properties is used in the analytical model to define both theoretical plots of Fig. 9. In addition, a direct characterization of the $d_{33}$ coefficient [21] gives a value of $275.4 \mathrm{pC} / \mathrm{N}$ of the thin-film actuators, concurring with the estimated $d_{31}$ coefficient.

The membrane strain demonstrates an average uniformity percentage of $16 \%$, calculated as the ratio of the standard deviation of the membrane strain to the average membrane strain, over 40 measurements in different regions of the grating. This is shown in Fig. 10, with the error bars depicting the maximum and minimum values. The uniformity variation is due to membrane thickness variation and anchor asymmetry in the double-anchored structure after membrane release. This mechanical nonuniformity is averaged out in the diffracted optical spectrum, although there is a corresponding spatial spreading of the energy in each diffracted order. In designing specific optical systems, therefore, the angle of acceptance should be sufficiently large so that the bulk of the diffracted order of interest is captured.

The PZT polarization-electric field hysteresis response, due to domain reorientations, is observed during $-10 \mathrm{~V}$ to $+10 \mathrm{~V}$ actuation, with a nonzero membrane displacement at $0 \mathrm{~V}$. The result matches the PZT film electrical characterizations. For compensating hysteresis and piezoelectric strain drifts, an open-loop drive signal could be modulated with a peak voltage pulse to saturate the PZT before actuation, or a closed-loop feedback could be implemented to continuously track the grating displacement. The modulated open-loop drive technique is postulated to be sustainable up to $10^{9}$ cycles, given related experience with open-loop piezoelectric cantilevers

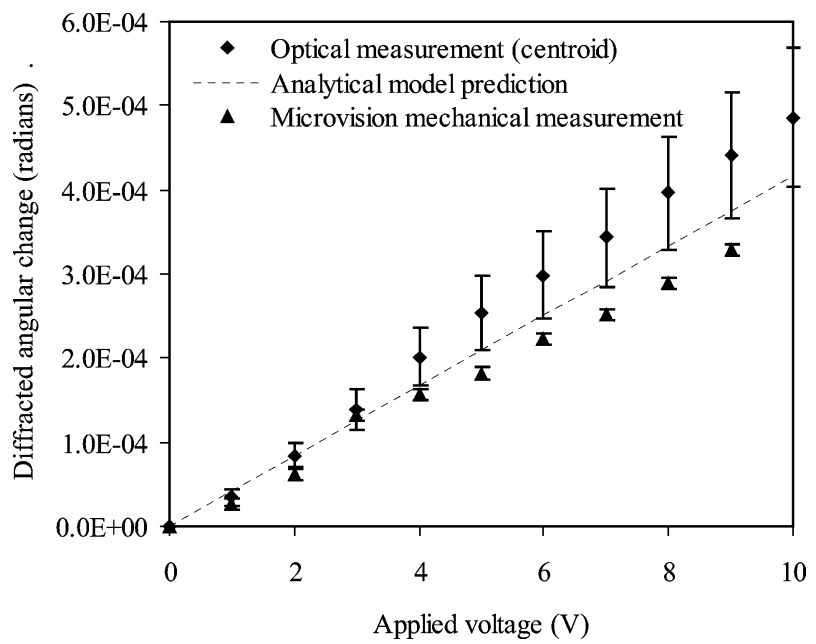

Fig. 11. First-order diffracted angular change against applied voltage obtained by optical image centroid processing and mechanical motion measurements. The optical measurement is corrected for tilt in the membrane through the finite-element mechanical model and is the main source of uncertainty.

driven up to $0.5 \times 10^{10}$ cycles without any observation degradation in performance or mechanical failure [15]. In addition, the frequency response of the double-anchored membrane is measured with a laser vibrometer and shows first mode resonance at $14 \mathrm{kHz}$, in agreement with theory. At the expense of tuning range, the dynamic response can be designed for higher operating frequencies by increasing the membrane stiffness of the lowest resonant modes.

\section{B. Optical Characterization of Diffracted Angular Change}

Measurement of the diffraction angular change was performed by imaging the first diffracted order onto a charge-coupled device (CCD) camera. A 632.8-nm HeNe laser was utilized as the illumination source and the diffracted order image centroid was analyzed for various actuation voltages. Fig. 11 shows the angular change effected on the first diffracted order when a voltage is applied to one of the two device actuators, for a device with Design I parameters. This angular change is then corrected for tilt in the membrane, via the matched finite-element model described in Section II, with only one of the two actuators used. Comparisons between the optical image centroid processing, mechanical membrane deformation measurements, and theoretical predictions show agreement within $15 \%$, limited by uncertainty in membrane tilt when actuated. At $10 \mathrm{~V}$, the angular change is estimated at $486 \mu \mathrm{rads}$ for this device. A device of Design II shows a correspondingly scaled diffracted angular change of $154 \mu \mathrm{rads}$ at $10 \mathrm{~V}$.

\section{CONCLUSION}

We have designed, fabricated, and characterized a piezoelectric-actuated analog tunable grating, using the controlled deformation of a supporting membrane. The design permits displacement resolution below a nanometer for the grating period change, a tunable range up to several nanometers (corresponding to up to $\sim 400 \mu \mathrm{rads}$ in the diffracted angular change), and minimizes out-of-plane motion of the membrane. 
Device fabrication integrates both surface and bulk micromachining to demonstrate process viability. The fabricated piezoelectric film shows a measured dielectric constant of 1200 and a derived $d_{31}$ coefficient of $-100 \mathrm{pC} / \mathrm{N}$. Mechanical measurements of the actuated device show grating period changes of $8.3 \mathrm{~nm}$ at $9 \mathrm{~V}$ and $4.9 \mathrm{~nm}$ at $10 \mathrm{~V}$ for two different device designs. For each design, the results agree well with our analytical and finite-element models. A diffraction angular change up to $486 \mu$ radians is measured at $10 \mathrm{~V}$ actuation, when corrected for membrane tilt in the matched finite-element modeling.

For comparison with a specific application, such as active grating compensation against thermal environment variations, our demonstration permits significantly lower power consumption (30 $\mathrm{nW}$ against order of $10 \mathrm{~mW}$ for integrated thermooptics), and finer control of tunability than thermal tuning. The $14 \mathrm{kHz}$ piezoelectric-actuated deformable membrane response time is applicable toward compensation for temperature variations in the environment, while faster response times can be considered for future device iterations. The device needs to be packaged against humidity in the environment to prevent electrical leakage in the long term. Future design iterations will reduce the asymmetrical rotation, tilt and bending of the membrane to improve the resolution in grating period change. Control and matching of residual stress in a low-stress silicon nitride diffusion barrier would reduce bow and out-of-plane motion in the membrane. To further reduce membrane bending, the membrane thickness could be increased for a cubic increase in bending stiffness, while trading-off a linear decrease in axial stiffness in the small-deflection regime. While specified in the context of active grating compensation against thermal environment fluctuations, this demonstration of analog tunability on the fundamental diffractive optical element anticipates implementations toward optical telecommunication applications and miniaturized spectrometers.

\section{ACKNOWLEDGMENT}

The authors would like to thank S. Desai and D. Freeman for the characterizations with the Computer Microvision system, L. Steyn for laser vibrometer measurements, and D.-G. Kim for direct $d_{33}$ evaluations. They also appreciate the helpful discussions with W. Liu, K. Broderick, M. Schmidt, A. Sinha, W.-C. Shih, G. Nielson, and C. Hidrovo.

\section{REFERENCES}

[1] G. B. Hocker, D. Youngner, E. Deutsch, A. Volpicelli, S. Senturia, M. Butler, M. Sinclair, T. Plowman, and A. J. Ricco, "The Polychromator: a programmable MEMS diffraction grating for synthetic spectra," in Proc. Tech. Digest of Solid-State Sensors and Actuators Workshop, Transducers Research Foundation, Cleveland Heights, OH, 2000, pp. 89-92.

[2] R. B. Apte, F. Sandejas, W. Banyai, and D. Bloom, "Grating light valves for high resolution displays," in Proc. Tech. Digest of Solid-State Sensors and Actuators Workshop, Transducers Research Foundation, Cleveland Heights, OH, 1994, pp. 1-6.

[3] J. Castracane, M. A. Gutin, and O. N. Gutin, "Micro-mechanically controlled diffraction: a new tool for spectroscopy," in Proc. SPIE, Diffractive/Holographic Technologies and Spatial Light Modulators, , vol. 3951, 2000, pp. 36-45.

[4] D. P. Resler, D. S. Hobbs, R. C. Sharp, L. J. Friedman, and T. A. Dorschner, "High-efficiency liquid-crystal optical phased-array beam steering," Opt. Lett., vol. 21, no. 9, pp. 689-691, 1996.
[5] D. E. Sene, J. W. Grantham, V. M. Bright, and J. H. Comtois, "Development and characterization of micro-mechanical gratings for optical modulation," in Proc. IEEE Micro Electro Mechanical Systems Workshop, Piscataway, NJ, 1996, pp. 222-227.

[6] X. M. Zhang and A. Q. Liu, "A MEMS pitch-tunable grating add/drop multiplexers," in Proc. IEEE Optical MEMS 2000 IEEE/LEOS International Conference, Piscataway, NJ, 2000, pp. 25-26.

[7] B. E. A. Saleh and M. C. Teich, Fundamentals of Photonics, 1st ed. New York: Wiley-Interscience, 1991, pp. 799-831.

[8] S. Jin, H. Mavoori, R. P. Espindola, L. E. Adams, and T. A. Strasser, "Magnetically tunable fiber Bragg gratings," in Proc. Tech. Digest of Optical Fiber Communication Conference, 1999, paper ThJ2-1.

[9] T. Storgaard-Larsen, S. Bouwstra, and O. Leistiko, "Opto-mechanical accelerometer based on strain sensing by a Bragg grating in a planar waveguide," Sens. Actuators A, Phys., vol. 52, pp. 25-32, 1996.

[10] D. M. Burns and V. M. Bright, "Micro-electro-mechanical variable blaze gratings," in Proc. IEEE MEMS Workshop, Piscataway, NJ, 1997, pp. 55-60.

[11] C. W. Wong, W.-C. Shih, Y. B. Jeon, S. Desai, D. Freeman, S.-G. Kim, and G. Barbastathis, "Analog tunable gratings with nanometer resolution," in Proc. Tech. Digest of Solid-State Sensors, Actuators, and Microsystems Workshop, Transducers Research Foundation, Cleveland Heights, OH, 2002, pp. 342-345.

[12] W.-C. Shih, C. W. Wong, Y. B. Jeon, S.-G. Kim, and G. Barbastathis, "Electrostatic and piezoelectric analog tunable diffractive gratings," in Proc. Conference on Lasers and Electro-Optics/Quantum Electronics and Lasers Science Conference, vol. 73, OSA Trends in Optics and Photonics, Washington, DC, 2002, paper CMP4.

[13] M. S. Weinberg, "Working equations for piezoelectric actuators and sensors," J. Microelectromech. Syst., vol. 8, no. 4, pp. 529-533, 1999.

[14] S. D. Senturia, Microsystem Design, 1st ed. Norwell, MA: Kluwer Academic, 2001, pp. 219-222.

[15] S.-G. Kim and K.-H. Hwang, "Thin-film micromirror array (TMA) for large information display systems," J. Soc. Inform. Display, vol. 8, no. 2, pp. 177-181, 2000.

[16] J. W. Goodman, Introduction to Fourier Optics, 2nd ed. New York: McGraw-Hill, 1996, pp. 73-83.

[17] W. Liu, J. S. Ko, and W. Zhu, "Preparation and properties of multiplayer $\mathrm{Pb}(\mathrm{Zr}, \mathrm{Ti}) \mathrm{O} 3 / \mathrm{PbTiO} 3$ thin films for pyroelectric application," Thin Solid Films, vol. 371, no. 1-2, pp. 254-258, 2000.

[18] C. W. Wong, "Strain-Tuning of Periodic Optical Devices: Tunable Gratings and Photonic Crystals," Sc.D. dissertation, Department of Mechanical Engineering, Massachusetts Institute of Technology, 2003.

[19] D. A. Pommet, E. B. Grann, and M. G. Moharam, "Effects of process errors on the diffraction characteristics of binary dielectric gratings," Appl. Opt., vol. 34, no. 14, pp. 2430-2435, 1995.

[20] D. M. Freeman, A. J. Aranyosi, M. J. Gordon, and S. S. Hong, "Multidimensional motion analysis of MEMS using computer microvision," in Proc. Tech. Digest of Solid-State Sensors and Actuators Workshop, Transducers Research Foundation, Cleveland Heights, OH, 1998, pp. 150-155.

[21] D.-G. Kim and H.-G. Kim, "Piezoelectric properties of lead zirconate titanate thin films characterized by the pneumatic loading method," Integr. Ferroelect., vol. 24, pp. 107-119, 1999.

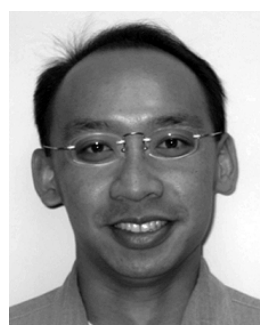

optics and photonics.

Dr. Wong is a Member of the American Physics Society (APS), the American Society of Mechanical Engineers (ASME), the Optical Society of America (OSA), and Sigma Xi.

Chee Wei Wong (M'03) received the B.S. degree in mechanical engineering and the B.A. degree in economics concurrently from the University of California at Berkeley in 1999. He received the M.S. and Sc.D. degrees from the Massachusetts Institute of Technology (MIT), Cambridge, in 2001 and 2003, respectively.

He was a Postdoctoral Associate at the MIT Microphotonics Center in 2003. He joined the faculty at Columbia University, New York, NY, in 2004 , with specific interests on micro- and nanoscale 


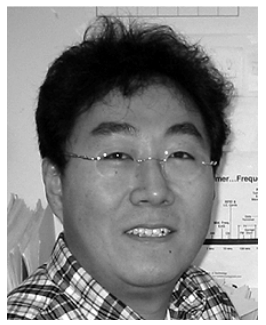

Yongbae Jeon received the B.S. and M.S. degrees in ceramic engineering from Yonsei University, South Korea, in 1988 and 1993, respectively, and the $\mathrm{Ph} . \mathrm{D}$. degree in materials science and engineering from Korean Advanced Institute of Science and Technology (KAIST), South Korea, in 2001.

$\mathrm{He}$ is currently a Postdoctoral Associate in the Micro and Nano Systems Laboratory with Professor S.-G. Kim in the Mechanical Engineering Department at the Massachusetts Institute of Technology (MIT), Cambridge. He had worked for developing Thin film Micro mirror Array (TMA) display system using piezoelectric MEMS technology at Daewoo Electronics Co. as a Senior Research Engineer from 1992 to 1997. He joined the research staff at MIT in April 2001 as Postdoctoral Associate of Mechanical Engineering. He has spent his professional career equally in academia and industry and has extensive experience in systems design of information display products, micromirror-array-based optical MEMS devices. His current research interests are in nanomanufacturing systems, optical tunable devices, large strain piezoelectric microactuators for $\mathrm{RF}$ switch, and piezoelectric micropower generation.

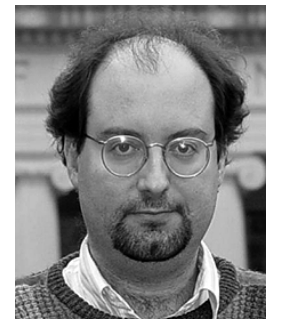

George Barbastathis (M'03) was born in Athens, Greece, on January 20,1971. He received the Electrical and Computer Engineer's degree from the $\mathrm{Na}-$ tional Technical University of Athens in April 1993 and the M.Sc. and Ph.D. degrees in electrical engineering from the California Institute of Technology (Caltech), Pasadena, in June 1994 and October 1997, respectively.

After Postdoctoral work at the University of Illinois, Urbana-Champaign, he joined the faculty at the Massachusetts Institute of Technology (MIT), Cambridge, in March 1999, where he is currently the Esther and Harold E. Edgerton Assistant Professor in the Mechanical Engineering Department. His research interests are in holography, optical MEMS, and nanostructured origami for 3-D optical sensing, interconnects and nanofabrication systems.

Dr. Barbastathis is a Member of the Optical Society of America (OSA), and he currently serves as Topical Editor for the Journal of the Optical Society of America A (Vision and Image Science).

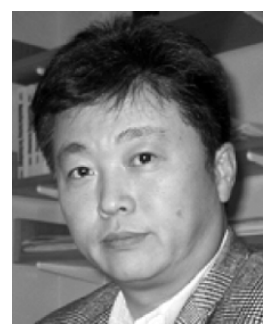

Sang-Gook Kim (M'03) received the Ph.D. degree in mechanical engineering from the Massachusetts Institute of Technology (MIT), Cambridge, in 1985.

In 2000, he joined the faculty of MIT as Associate Professor of Mechanical Engineering. He has spent his professional career equally in academia and industry and has extensive experience in systems design of multiscale products. His current research interests are in PZT MEMS for optical/RF switching, optical packaging and active alignment, micropower generation and energy harvesting, carbon nanotube assembly, and nanomanufacturing.

Dr. Kim is a Member of the American Society of Mechanical Engineers (ASME), and a candidate member of the National Academy of Engineering of Korea and the International Institution for Production Engineering Research (CIRP). 